\title{
Designing and fabrication of a nozzle to reduce the consumption of water
}

\author{
Adham Abdu \\ Dr. Basim Khidhir
}

\author{
MIDDLE EAST COLLEGE \\ Middle East College
}

Reducing the consumption of water is very important in today's world. Around 1.8 million people die every year due to lack of clean water. There are different ways to reduce the consumption of water in different aspects of daily life. This project report will be mainly dealing with the designing or fabrication of an already existing nozzle to conserve water from the tap waters. This report is an experimental study on comparison of existing nozzle with the designed nozzles in this paper. This is also a live project with Majid al Futtaim consultancy, MCC (Muscat city Centre). Different designs were made for this study.

\section{Introduction}

Water is one of the most important substance on earth. All the creatures on the earth needs water to survive. If there was no water on the earth, then there would be no life on it. There are so many other uses of water to us. Water is used in many ways throughout our lives, like for cooking, hygiene purposes, for plants or gardens etc.

There are so many issues in this world due to lack of water like deadly diarrheal diseases, cholera, typhoid etc. If only a small rate of water is conserved in a daily basis, it would be of a very good value for any county and its future. The main objective of this project is to investigate on an upcoming or already existing problem in a wider range and to find an applicable solution for it.

There are so many counties in our world even without clean water. Almost 10,000 people die from lack of clean water every day (industry tap, 2014). Lack of water also affects every continent and around 2.8 billion people around the world at least one month out of every year. Overall more than 1.2 billion people lack access to clean drinking water (science daily, 2018). 2,000 children under the age of five die every day. 783 million people do not have clean water (global citizen, 2012).

This happens mostly because these people stay in remote areas and their water sources are far away from their residential areas. Even if they were to go to these water sources of theirs, it would not be affordable for them and the water would be unclean. Even if they wanted to go get this unclean water, they would have to walk for a lifetime. Which means they won't have time for anything including school. And consumption of unclean water will lead to diseases like diarrheal, cholera, typhoid, waterborne diseases, guinea worm disease, dysentery etc. If they don't have enough water for drinking, it's obvious that they don't have water for growing food, which will make them hungry also and give them more sickness. Diseases due to lack of food are chronic diseases such as cardiovascular disease, hypertension, cancer, diabetes mellitus etc. The problems get worse and worse just due to lack of water. All of these will lead them to poverty.

Since caution is better than cure, it is our duty to be alert on to such issues. This project would be a preparation or preplanning to these kinds of problems.

"There are many reasons why water is frequently referred to as "the new oil." About $71 \%$ of the earth's surface is covered with water, but that doesn't mean this unique, life-sustaining substance is in abundant supply. The U.S. at 494,000 gallons $(1,870$ cubic meters $)$ per year or 100 gallons (.38 cubic meters) per day per capita, tops the list, with Canada second at 


\section{Journal of Student Research}

Fourth Middle East College Student Research Conference, Muscat, Sultanate of Oman

388,300 gallons $(1,470$ cubic meters) per year. In the U.S., several large areas in the Southeast, Southwest and West are characterized as suffering severe, extreme or - worst case -exceptional drought conditions."

There has been drastic increase on how the water authority raised their price on water, from last year, the average cost of water has risen $7.3 \%$. From since 2013, the average water cost has been increased to nearly $30 \%$. The cost of 1000 gallons or 3.8 kiloliters including sewer charges are from $3 \$-7$ USD (1.5 OMR - 2.69 OMR).

This project is based on the wastage and exhaustion of fresh water from the face of the earth. In the coming days, things are going to get worse. Because we are not learning from our mistakes. Carbon emissions are keeping on increasing and we are doing nothing about it. The earth's temperature is increase by each century. From $19^{\text {th }}$ century the earth's temperature has increased to $1.5^{\circ} \mathrm{C}$ till now. At $2^{\circ} \mathrm{C}$ the urban population will be exposed to severe water shortage, which might happen in our near future. And these urban shortages would lead to lack of food due to lack of water for agricultural purposes.

The conservation of water is one of the most important things which should have been looked on to in the past itself, because of the depletion of fresh water day by day. If the governments and the authorities are still not awaking and finding solutions to this problem, it would not only put us on danger but also the many more future generations to come.

Simply by fixing leaks many of the water issues in today's world can be reduced. There has been report which says if the leakages of the pipes are taken care of almost $14 \%$ of water could be consumed. Sub-meters can be installed to detect leaks. Replacing pipes with drilled holes with spray manifolds which will cut the fat of water and serve the purpose. Pipes with holes use excessive amounts of water be sure the proper nozzles are being used for the application. Install high-pressure, low-volume nozzles on spray washers.

\section{Literature review}

\section{Energy efficient cutting fluid supply: The impact of nozzle design}

Cutting fluids are used in grinding operations for great effect and help maintain high productivity in these processes. Pumping performance and nozzle design are crucial for this.

There are different nozzle types available-each with its own set of benefits or drawbacks.

The categories are created based on the function, focusing and nozzle geometry. Equation based models have been applied in this article to determine the energy savings of different designs. The researches carried out different experiments to determine the effect of nozzle and parameters of the process on the efficiency. It was concluded that useful flow rate (through the grinding gap) is influenced by nozzle design, position and fluid speed. The choosing of a suitable nozzle for a specific purpose saves about $23.5 \%$ of the pump's power consumption. Further parameters need to be analyzed to extend the scope of the study.

\section{Fabrication of micro glass nozzle for micro droplet jetting}

The most essential part of the micro-drop generating technique is an ejection aperture nozzle. The main factors which effects the ejection capacity are the size of its diameter, the geometry flow channel and the impedance of the fluid. Each one of the applications mentioned requires an ideal size and shape for the micro nozzles. Several methods are used for making the micro nozzles such as laser drilling, micromechanical drilling, electron beam machine, electroforming over a sacrificial 


\section{Journal of Student Research}

Fourth Middle East College Student Research Conference, Muscat, Sultanate of Oman

post etc. overall this article is based on the low-cost fabrication process of micro glass nozzle which includes four steps for its development.

The material used for the manufacturing of the glass micro-nozzle is borosilicate glass pipette, the reason for using this material is due to its various properties, there are advantages such as a) low fluid impedance and smooth surface b) the chemical stability is excellent as well as its resistance to the alkali corrosion except for the hydrofluoric acid c) makes the process easy due to its softening point application d) the optical characteristics are good which makes this material suitable for generating the micro-glass nozzle and e) its durability and low cost. These properties overall make it best suited for the manufacturing and performing the experiments.

The fabrication of the micro-glass nozzle involves four steps which are a) First Heating, in this step the pipette of the glass is heated and melted above its softening point and then it is pulled this is done to attain the sharp cone of the glass tube b) Second Heating, in this step it is made sure that the flow channel is shortened for making the flow resistance less and it also confirms that the direction of the injecting it is right c) Fine Grind, the second last step ensures the diameter of the hole of the micro-glass is as desired and it is checked under the microscope since it's a very delicate operation to grind the glass and d) Post Heating is the final step which involves heating of the glass nozzle tip in order to obtain smooth polished surface and edges with no sharpness being left and hence the end result is obtained.

After the completion of this process they held an experiment to observe the results by comparing the micro-glass nozzle ejection process with the hydrophobic nozzle and non-hydrophobic nozzle however it was concluded that the wetting the surface of nozzle does affect the operation of generating of droplet. The final conclusion to this articles content was that the micro-glass nozzle obtained is a low-cost fabricated nozzle and the manufacturing of the nozzle takes very less time, it has a very important step in the completion of a project. In order to complete a project, the process of methodology good repeatability also has accuracy in its size and the size and shape can be changed as desired during the making process hence it is a very easy and less time-consuming process with finest results.

\section{Methodology}

Methodology must be completed, without collecting the data and analyzing the result it's impossible to proceed hence, collection of data and its analysis is known as methodology. The main purpose of this project is fabrication of different type of nozzle to reduce the consumption of water. There are two ways in which the data was gathered first is the Primary source and second is the Secondary source.

The primary source is the data obtained from a direct source and is something which is not obtained from someone else's observation. It is always observed and gathered directly, this firsthand information is called primary source. For example, Questionnaire, Interview and observational study. For the completion of my project a lot of research had to be done on the fabrication of nozzle in different industries in order to have a comparison, the results were analyzed based on the reduction of water wastage.

a) Firstly a complete study was done to have a better understanding of the topic and have a clear idea on what is to be focused and what could be the challenges faced during the project for which the lecturers specialized in this field were consulted, who helped me a lot to gather the required information and focus.

b) After gathering required knowledge, Majid Al Futtaim Consultancy which is an organization who had done a similar project for the Muscat City Centre (MCC) was consulted to gather a complete information. 


\section{Journal of Student Research}

Fourth Middle East College Student Research Conference, Muscat, Sultanate of Oman

c) The dimensions of the MCC nozzle was collected with the help of Vernier caliper from the MCC wash basins.

d) After finding the perfect plan it was focused on how the implementation of different types of nozzles have helped them and what changes were observed before and after the execution, as the positive results could help me reduce the wastage of water.

Secondary source is a way of gathering information's which are second handed or maybe used previously. For example, Journal articles, Text books, bibliography, online websites and articles, videos, dictionaries etc. a lot of website and journal articles such as Design and Fabrication of Miniature Supersonic Nozzles, Change the way you spray to maximize water conservation and many more were referred.

a) An interview was also conducted with Mr. Sujith the Operational Supervisor of the Muscat City Centre, where the project was implemented earlier by the Majid Al Futtaim Consultancy. The interview helped a lot in gathering data and pre-analyzing the positive results which could be achieved on completion of this project.

b) When the information was gathered about the Thermo Fluids and the types of nozzles which are already implemented, it was focused on designing and creating new designs of the nozzles which will be beneficial functionally. Frequent meetings were held with the Majid Al Futtaim Consultancy as similar project was carried out by them. The nozzle pictures and videos were taken as samples for reference and modified to a new version.

\section{Data Analysis}

The Data analysis is an important part of the methodology process. In this step, all the data and information that was collected is put together in order to analyze and obtain results. After getting all theoretical information and consulting the organization it was clear that the project will be beneficial to the environment. According to the examined results of Muscat City Centre there was a difference in the consumption of water after the implementation of different nozzle. The consumption of water was determined using a Variable Frequency Drive (VFD) which is a device used for controlling the pressure and flow of the fluid as per demand. It works by controlling the frequency of electrical power supplied to the water pump, hence the VFD makes it simpler to obtain the required results.

\section{Numerical}

The theoretical results are important because of the comparison purposes. These results are obtained from the given theories. These results can be taken as the expected results for the experiments or simulations.

\section{Equations}

Different equations like Bernoulli's equation and continuity equation where used to determine the fluid flow, the sum of pressure, potential and kinetic energies etc.

$$
\mathrm{p}+1 / 2 \mathrm{pV} 2+\mathrm{pgh}=\mathrm{constant}
$$

Where, $\mathrm{P}=$ Pressure, $\rho=$ density, $\mathrm{V}=$ velocity, $\mathrm{h}=$ elevation, $\mathrm{g}=$ acceleration due to gravity.

$$
\mathrm{pA} 1 \mathrm{~V} 1=\mathrm{pA} 2 \mathrm{~V} 2
$$




\section{Journal of Student Research}

Where, $\mathrm{A}=$ the cross-sectional area of flow, $\mathrm{V}=$ the mean velocity, $\rho=$ density

\section{Final design}

Different types of designs were produced by manual drawing and auto cad. From all these different ideas of designs combining a final design and the already existing mcc nozzle design was created in the ANSYS 2019 R1 software to compare its capabilities.

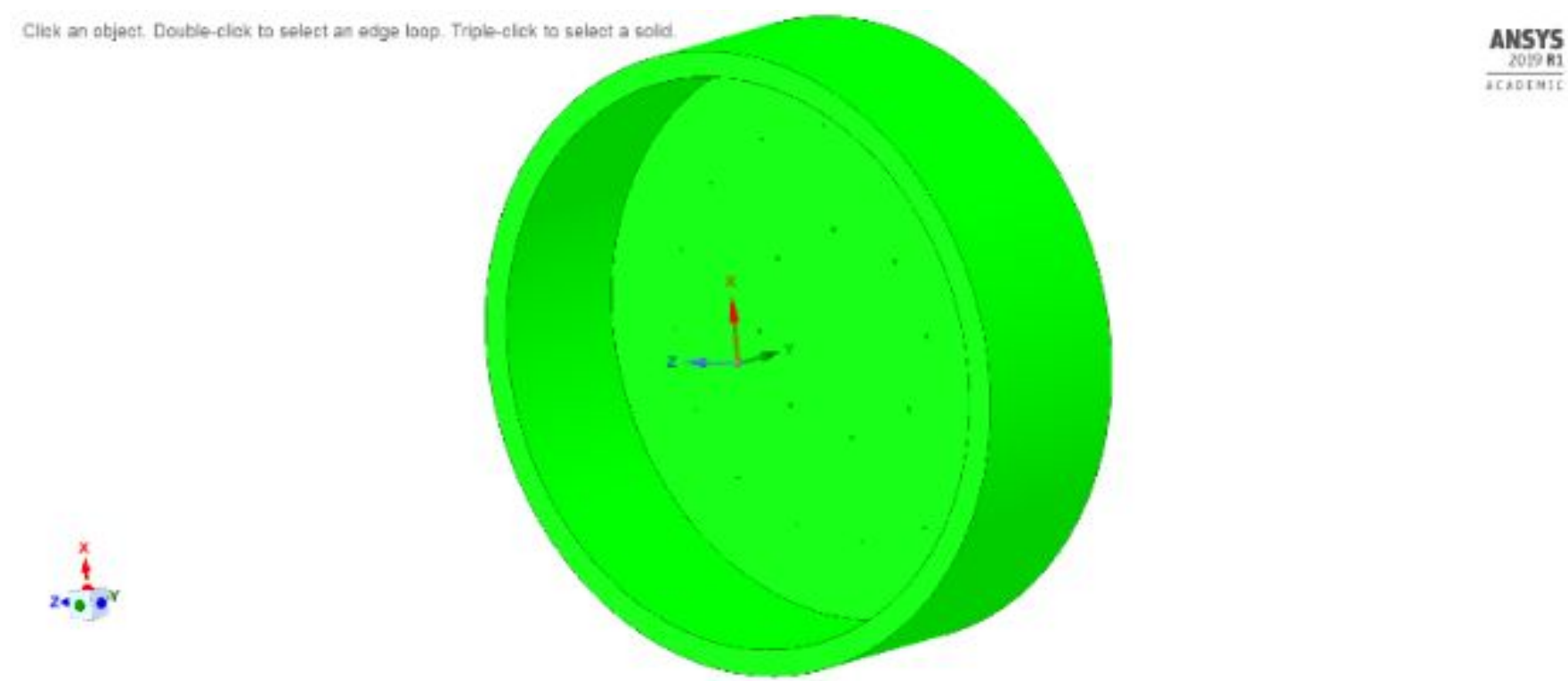

Figure 1. Already existing MCC nozzle

Cirk an cbject Double-click to select an edge loop. Triple-cirk to select a sold.

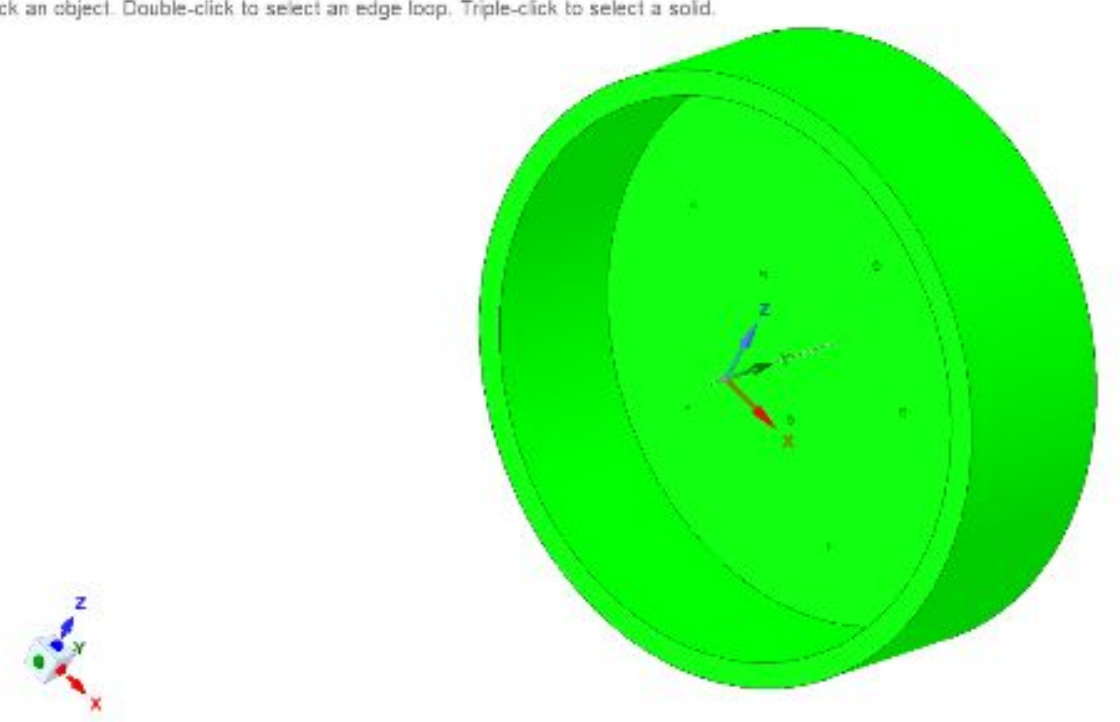

Figure 2. Fabricated nozzle

Through different procedures in ANSYS 2019 R1 software the nozzles were compared and analyzed. The different steps in ANSYS was space claim, meshing, setup and checking, solutions, results and simulations.

\section{Results and simulations}


There has been different animations of results or simulations of water flow through the nozzles which are shown below. All these results are in form of animation movies which shows the movement of the fluid from the inlet to the outlet and its different intensity of velocities at different places.

\section{Simulation of the already existing nozzle in MCC}

The nozzle holes with the red lines coming out from the outlet are the holes which will be having the most fluid flow from, it is also the nozzle hole from which the velocity of the fluid flow is the most.

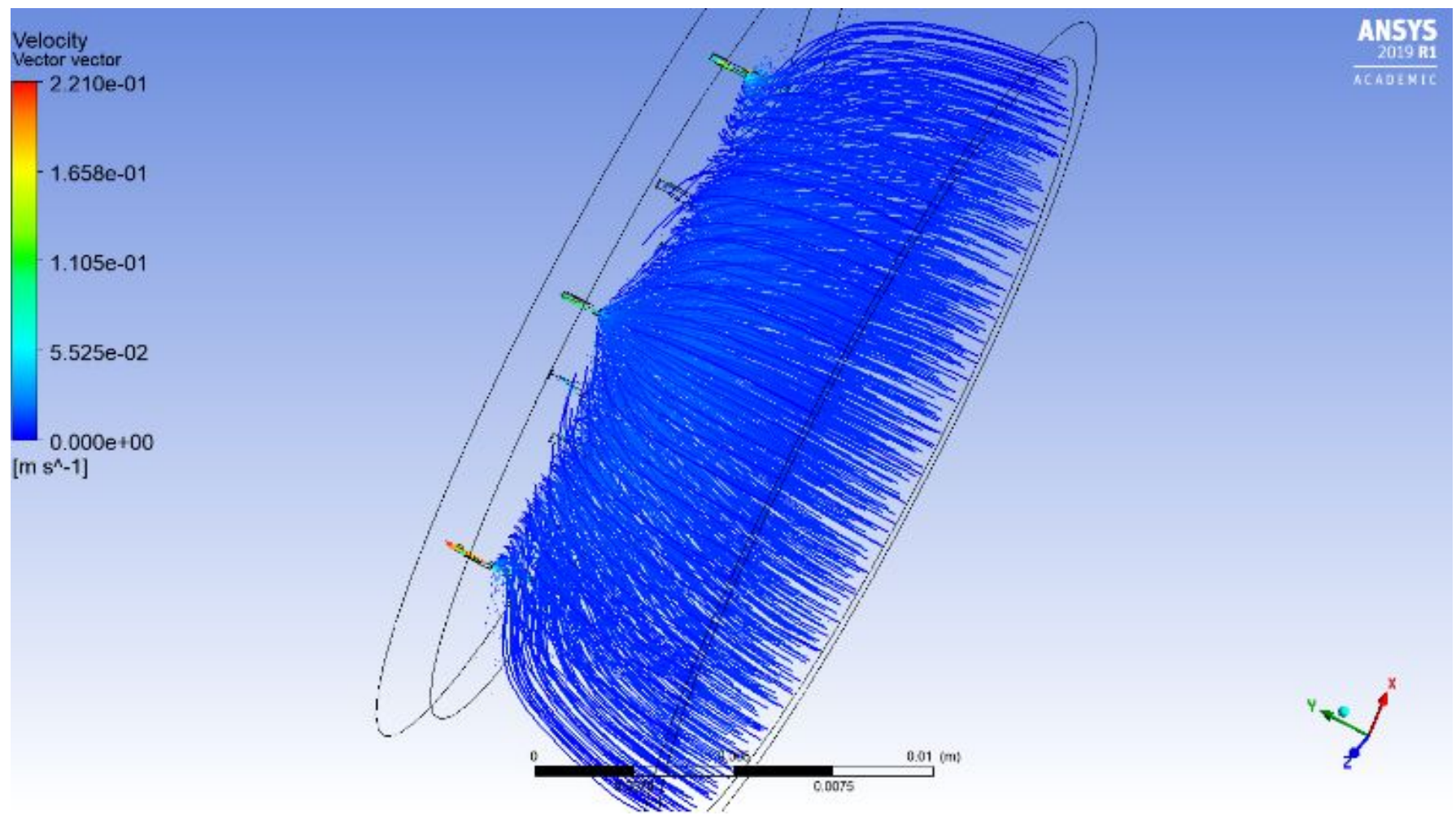

Figure 3. Continuous fluid flow in the nozzle (MCC nozzle)

The above shown is the continuous fluid flow inside the nozzle. The water flow keeps on continuing in the animation $\infty$ times. The water flow can be set in the options menu. From, the animation part it's clear that the central part or the center holes of the outlet nozzle ejects water first and then the respectively till the far end nozzle holes, away from the center. The water which does not get ejection holes bounces back and forms a circular pattern, which is again taken away by the continues fluid coming. 


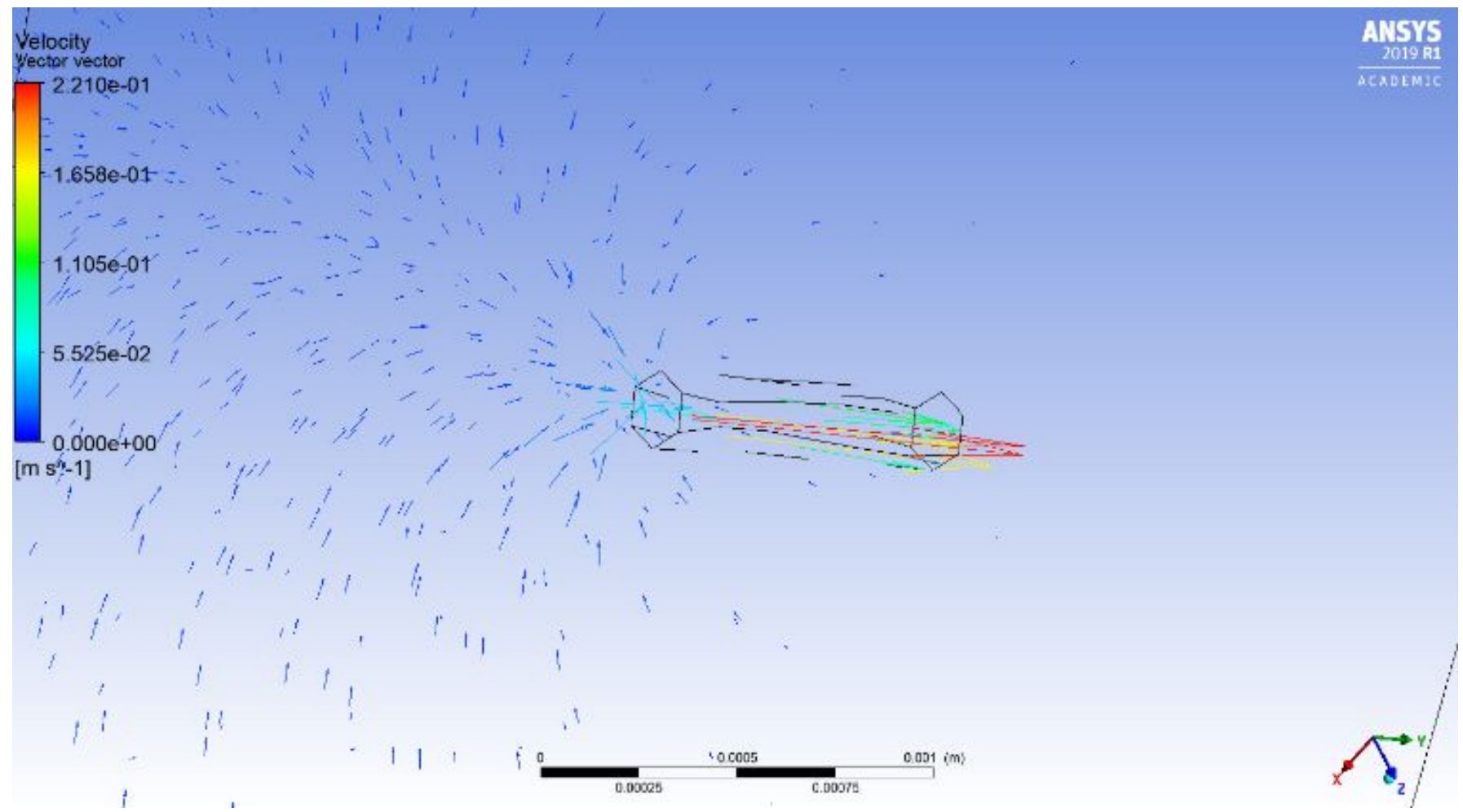

Figure 4. Velocity of fluid in an ejection hole $\left(1^{\text {st }}\right.$ case)

Here, in the above picture of the nozzle outlet simulation, it shows the exact velocities of the fluid in nozzle outlets. Different colors are represented to specify the intensity of velocity. The rear end of the nozzle hole is shown in the picture as light blue color. Ejection area inside the nozzle hole shows us a light green color. After the ejection area of the fluid shows yellow color and away from the nozzle hole is the red color given.

Each of these colors are having different specific velocities:

\begin{tabular}{|l|l|}
\hline Light blue & $5.525 \mathrm{e}-02$ \\
\hline Light green & $1.105 \mathrm{e}-01$ \\
\hline Yellow & $1.658 \mathrm{e}-01$ \\
\hline Red & $2.210 \mathrm{e}-01$ \\
\hline
\end{tabular}

Table 1. Velocities of different exit holes in already existing MCC nozzle

\section{Simulation of the fabricated nozzle}




\section{Journal of Student Research}

Fourth Middle East College Student Research Conference, Muscat, Sultanate of Oman

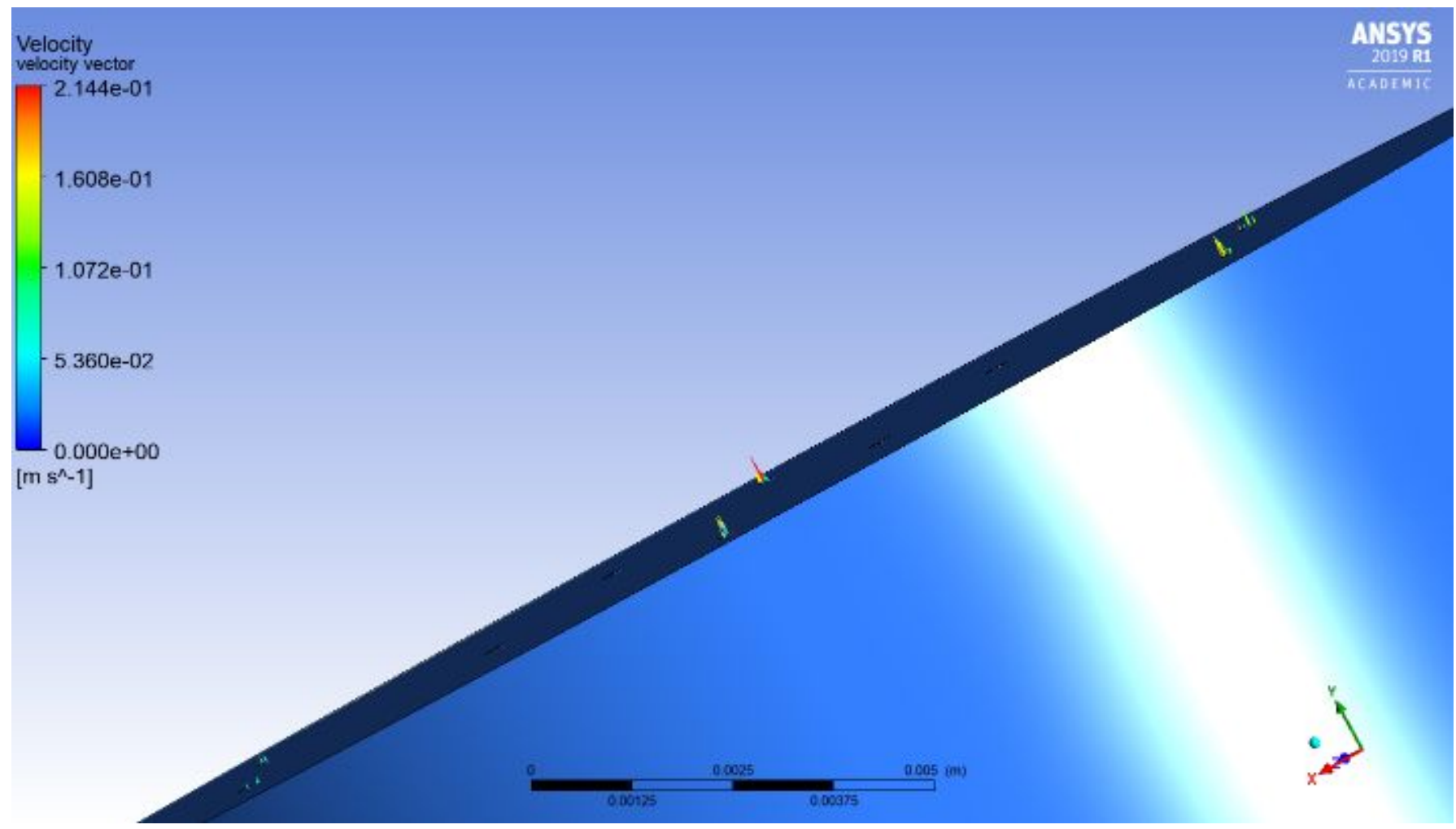

Figure 5. Fluid flow through the outlet of the nozzle

The nozzle holes with the red lines coming out from the outlet are the holes which will be having the most fluid flow from, it is also the nozzle hole from which the velocity of the fluid flow is the most.

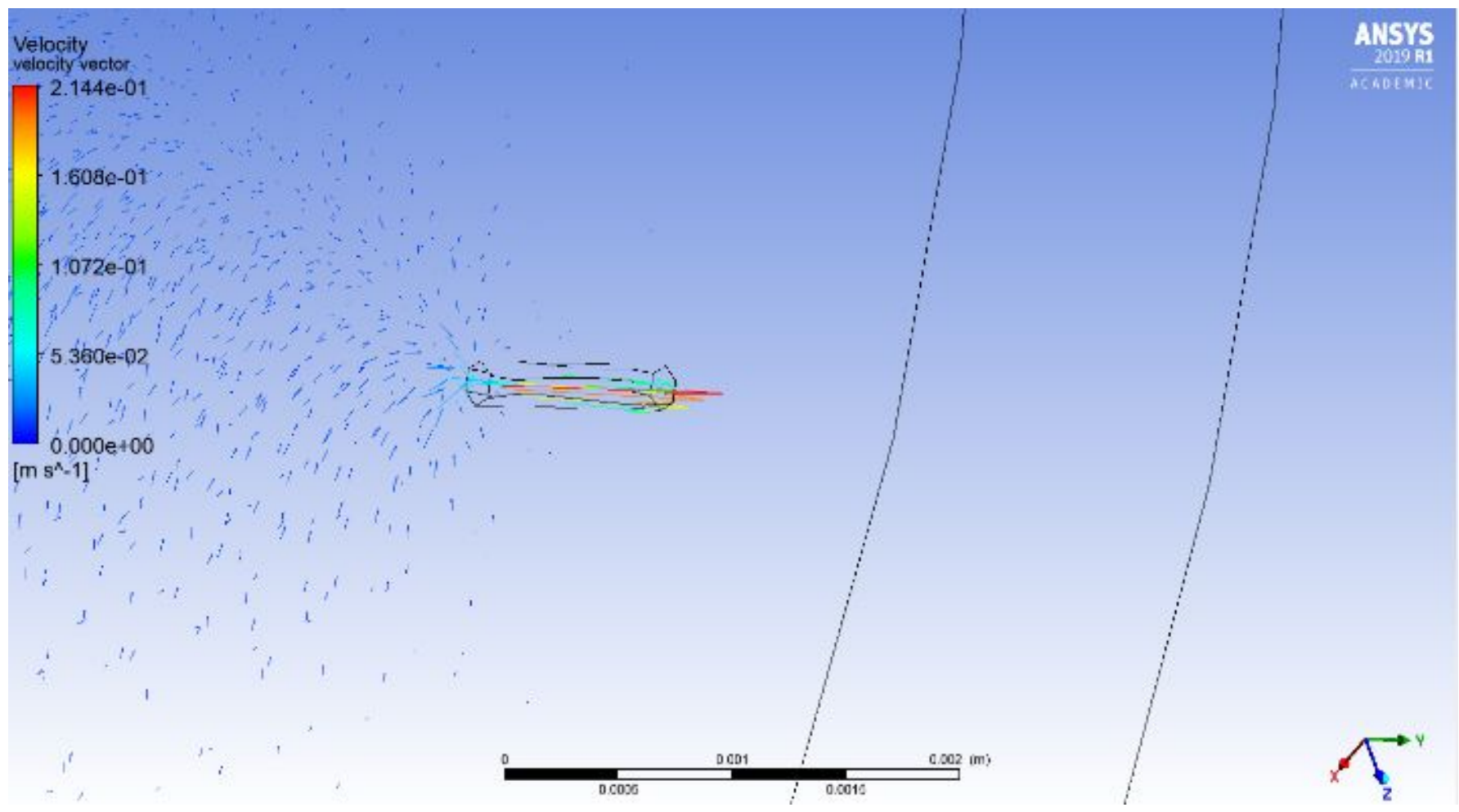

Figure 6. Velocity of fluid in ejection hole 
Here, in the above picture of the nozzle outlet simulation, it shows the exact velocities of the fluid in nozzle outlets. Different colors are represented to specify the intensity of velocity. The rear end of the nozzle hole is shown in the picture as light blue color. Ejection area inside the nozzle hole shows us a light green color. After the ejection area of the fluid shows yellow color and away from the nozzle hole is the red color given.

Each of these colors are having different specific velocities:

\begin{tabular}{|l|l|}
\hline Light blue & $5.536 \mathrm{e}-02$ \\
\hline Light green & $1.072 \mathrm{e}-01$ \\
\hline Yellow & $1.608 \mathrm{e}-01$ \\
\hline Red & $2.144 \mathrm{e}-01$ \\
\hline
\end{tabular}

Table 2. Velocities of different exit holes in fabricated nozzle

\section{Discussion}

\section{Comparing the results}

The results from both the simulations are nearly the same. The main difference is the velocity of water in different parts of the ejection holes.

\section{$\Delta \mathrm{V}=$ Velocity in each phase (MCC nozzle) - Velocity in each phase (fabricated nozzle)}

\begin{tabular}{|l|l|l|l|}
\hline Velocity range & 1st case $(\mathrm{V} 1)$ & 2nd case(V2) & $\Delta \mathrm{V}$ \\
\hline Light blue & $5.525 \mathrm{e}-02$ & $5.536 \mathrm{e}-02$ & $-1.10 \mathrm{E}-04$ \\
\hline Light green & $1.105 \mathrm{e}-01$ & $1.072 \mathrm{e}-01$ & $3.30 \mathrm{E}-03$ \\
\hline Yellow & $1.658 \mathrm{e}-01$ & $1.608 \mathrm{e}-01$ & $5.00 \mathrm{E}-03$ \\
\hline Red & $2.210 \mathrm{e}-01$ & $2.144 \mathrm{e}-01$ & $6.60 \mathrm{E}-03$ \\
\hline
\end{tabular}

Table 3. Comparison of the results of the MCC nozzle and fabricated nozzle.

The velocity difference is shown above in the column of $\Delta \mathrm{v}$. In the $1^{\text {st }}$ case, the velocity in the start of ejection hole increases from 5.525e-02 to $2.210 \mathrm{e}-01$ which is the velocity of fluid in the end of ejection hole. Whereas, in the $2^{\text {nd }}$ case, the velocity in the start of ejection hole increases from $5.536 \mathrm{e}-02$ to $2.144 \mathrm{e}-01$ which is the velocity of fluid in the end of ejection hole.

When we compare the velocities of both the simulations, it's clear that in the $1^{\text {st }}$ case, the initial ejection velocity is much lesser than that of the $2^{\text {nd }}$ case. Where as in the next 3 phases of velocities, $1^{\text {st }}$ simulation is having more velocity than that of the $2^{\text {nd }}$ simulation velocities.

Overall, in the $1^{\text {st }}$ case of simulation - the velocity from the inlet would be less and the velocity from the outlet would be more.

Whereas, in the $2^{\text {nd }}$ case of simulation - the velocity from the inlet would be more and the velocity from the outlet would be less

\section{Conclusion}

The before and after implementations of the existing nozzle was studies. The results were that the wastage of water saved is 10 times lesser than that of the tape water which dint have any nozzle fixed. Different types of designs were constructed in AutoCAD and ANSYS 2019. The best design out of all the designs were selected and programed for fabrication. The designed nozzle was then 
simulated in ANSYS 2019 R1 software, which helped in the study of performance for the designed nozzle.

\section{Acknowledgements}

The completion of this project was only possible because of the guidance and assistance of many individuals. Their support and supervision can never be forgotten, without which this project would not be possible. Firstly, thanking god almighty for all his blessings and for providing me with such wonderful supervisor and teachers. Really, thankful my Middle East college, for providing me with all the facilities which was necessary for my project. I am also thankful to Muscat city Centre for their cooperation and involvement in the project. Also would like to acknowledge mechanical lecturers, like Dr. Fiseha mekonnen guangul and Dr. Abdalellah Mohammed for their hard work in the making of this project. Any omission in this brief acknowledgement does not mean lack of gratitude.

\section{References}

Rico, J. A. S., Endo, G., Hirose, S. \& Yamada, H., 2017. Development of an actuation system based on water jet propulsion for a slim long-reach robot. 4(8), pp. 1-17.

Hansen, C. J., Saksena, R., Kolesky D. B., Vericella, J. J. (2013), 'High-Throughput Printing Via Microvascular Multi nozzle Arrays', Advanced Materials, 25, 96-102

Xie.D, Chang. X, Shu. X, Xiao. J, Zhang. H (2014), 'fabrication of micro glass nozzle for microdroplet jetting', hindawi publishing corporation, (15 July 2014)

Cooley w., Brockert p. (1972),'Design and fabrication of exponential nozzle', terraspace incorporated, (July 1972)

Chindambaran narsyanan, (2017), 'optimized abrasive waterjet nozzle design using genetic algorithms, (10 Jan 2017)

Asad Ahmed R, (2017), 'Design and fabrication of miniature supersonic nozzle', (26 March 2017) 